\title{
Clonal T cell receptor gene rearrangements in coeliac disease: implications for diagnosing refractory coeliac disease
}

\author{
Shafinaz Hussein, ${ }^{1}$ Tatyana Gindin, ${ }^{1}$ Stephen M Lagana, ${ }^{1}$ Carolina Arguelles-Grande, ${ }^{2}$ \\ Suneeta Krishnareddy, ${ }^{2}$ Bachir Alobeid, ${ }^{1}$ Suzanne K Lewis, ${ }^{2}$ Mahesh M Mansukhani, ${ }^{1}$ \\ Peter H R Green, ${ }^{2}$ Govind Bhagat ${ }^{1,2}$
}

\begin{abstract}
- Additional material is published online only. To view please visit the journal online (http://dx.doi.org/10.1136/ jclinpath-2018-205023)

'Department of Pathology and Cell Biology, New York Presbyterian Hospital/Columbia University Medical Center, New York, USA

${ }^{2}$ Department of Medicine, Celiac Disease Center, New York Presbyterian Hospital/Columbia University Medical Center, New York, USA
\end{abstract}

\section{Correspondence to}

Dr Govind Bhagat, Department of Pathology and Cell Biology, New York-Presbyterian Hospital, New York 10032, USA; gb96@ cumc.columbia.edu

SH and TG contributed equally.

Received 13 January 2018 Revised 28 March 2018

Accepted 29 March 2018 Published Online First 27 April 2018
Check for updates

To cite: Hussein $S$,

Gindin T, Lagana SM

et al. J Clin Pathol

2018:71:825-831.

\section{ABSTRACT}

Aims Refractory coeliac disease type II (RCDII), a rare complication of coeliac disease (CD) associated with high morbidity, requires identification of a clonal population of phenotypically aberrant intraepithelial lymphocytes (IELs) for diagnosis. However, data regarding the frequency and significance of clonal $\mathrm{T}$ cell receptor (TCR) gene rearrangements (TCR-GRS) in small bowel (SB) biopsies of patients without RCDII are limited.

Methods We analysed results of TCR-GR analyses performed on SB biopsies at our institution over a 3-year period, which were obtained from eight active CD, 172 CD on gluten-free diet (GFD), 33 RCDI, and three RCDII patients and 14 patients without CD. TCR-GR patterns were divided into clonal, polyclonal and prominent clonal peaks (PCPs) and these patterns were correlated with clinical and pathological features.

Results Clonal TCR-GR products were detected in biopsies from $67 \%$ of patients with RCDII, $17 \%$ of patients with RCDI and $6 \%$ of patients with GFD. PCPs were observed in all disease phases (range $12 \%-33 \%)$. There was no significant difference in the TCR-GR patterns between the non-RCDII disease categories $(p=0.39)$. A higher frequency of surface CD3(-) IELs was noted in cases with clonal TCR-GR, but the PCP pattern did not show associations with any clinical or pathological feature. Persistence of clonal or PCP pattern on repeat biopsy was seen for up to 2 years without evidence of RCDII.

Conclusions Clonal TCR-GRs are not infrequent in cases lacking features of RCDII, while PCPs are frequent in all disease phases. TCR-GR results should be assessed in conjunction with immunophenotypic, histological and clinical findings for appropriate diagnosis and classification of RCD.

\section{INTRODUCTION}

Coeliac disease (CD) is a common autoimmune disorder (prevalence $\sim 1 \%$ ), which occurs in genetically susceptible individuals who develop an immune reaction to gluten. The diagnosis of CD is currently based on the presence of small bowel (SB) mucosal abnormalities (villous atrophy (VA), crypt hyperplasia, increased intraepithelial lymphocytes (IELs) ${ }^{1}$ ) and seropositivity for antiendomysial (EMA) and/or antitissue transglutaminase (tTG) antibodies, which are highly sensitive and specific for the disease. Permissive genetics (human leucocyte antigen (HLA)-DQ2 or HLA-DQ8 alleles) support the diagnosis, whereas their absence virtually excludes CD. ${ }^{12}$

A gluten-free diet (GFD) is the mainstay of treatment, adherence to which results in the normalisation of SB histopathology and disappearance of disease-associated antibodies in most instances. Persistence of severe symptoms and VA despite strict GFD occurs in a small subset of patients who are deemed to have refractory $\mathrm{CD}$ (RCD). RCD is classified into RCDI and II, based on the absence or presence of a clonal IEL population manifesting an aberrant phenotype. ${ }^{134}$ The correct classification of RCD is critical, given that RCDI follows a relatively benign course, while RCDII is associated with poor prognosis, with approximately $50 \%$ of RCDII patients developing enteropathy associated $\mathrm{T}$ cell lymphoma (EATL) within 5 years. $^{5-7}$

Immunohistochemistry and flow cytometry (FC) are currently used to assess the phenotype of IELs. $\mathrm{FC}$ is more sensitive and specific in assessing the expression/loss of surface CD3, CD8 and T cell receptor (TCR) and aids in precise quantification of phenotypically aberrant IELs. ${ }^{6}$ The clonal nature of IELs is evaluated by TCR- $\gamma$ and/or TCR- $\beta$ gene rearrangement (TCR-GR) analyses; however, these assays can vary in sensitivity and/or specificity depending on the method used. ${ }^{9}{ }^{10}$ Clonal TCR-GRs are detected in the majority of RCDII cases; however, a few studies have documented clonal TCR-GRs in uncomplicated CD (up to 36\% of cases). These studies comprised a small number of patients, often lacking adequate clinical data, and many utilised older TCR- $\gamma$ GR assays with non-standardised PCR primers and protocols. ${ }^{911-14}$

Since determining the presence of a clonal population of phenotypically aberrant IELs is integral to the diagnosis and correct classification of RCD, we sought to evaluate the frequency of clonal TCR-GR in SB biopsy samples from a large cohort of patients with $\mathrm{CD}$ that had FC-defined IEL phenotypes, across different phases of $C D$, with a sensitive PCR assay using the BIOMED-2 primers. ${ }^{10}$ Associations between clonal TCR-GR results and SB histology, IEL phenotype, clinical presentation and serological findings were also assessed. 

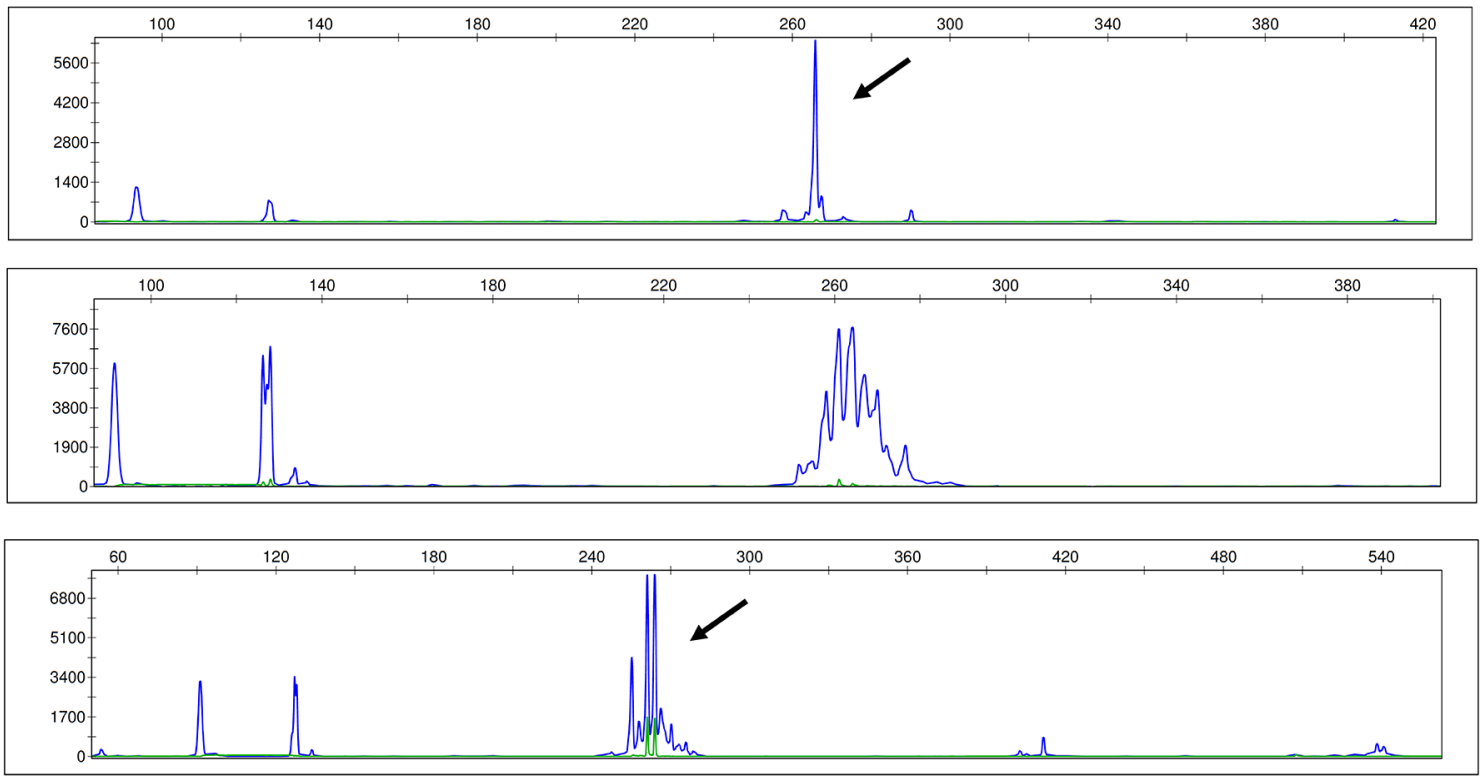

Figure 1 Representative images of clonal (top), polyclonal (middle) and prominent clonal peak (bottom) TCR-GR patterns. Arrows indicate distinct peaks.

\section{MATERIALS AND METHODS Case selection}

We identified SB biopsies from established and suspected patients with CD whose samples were submitted for TCR-GR analysis at our institution over a 3-year period. FC and SB histology, clinical features and laboratory findings, including disease duration, type of symptoms (typical/diarrhoea predominant vs atypical), therapy and serological abnormalities (antigliadin antibodies, deamidated gliadin peptide, EMA and tTG), as well as clinical outcomes were obtained.

\section{TCR- $\boldsymbol{\beta}$ GR analysis by fluorescent PCR}

DNA was extracted from fresh or formalin fixed paraffin embedded (FFPE) SB biopsies. Multiplexed fluorescent PCR analysis was performed using BIOMED-2 primers followed by high-resolution capillary electrophoresis. ${ }^{10}$ A clonal TCR-GR was defined as a prominent single peak twice the height of the second highest peak or 2.5 times the height of the third highest peak in cases with two prominent peaks within the expected size range. Polyclonal TCR-GR was defined as multiple peaks with a Gaussian peak-height distribution. Prominent clonal peaks (PCPs) were defined as discrete peaks that were much higher than the polyclonal background but did not meet the aforementioned 'peak-height-ratio' criteria for clonality (figure 1).

\section{Flow cytometry}

Four-colour FC of fresh SB biopsy samples was performed with FACSCalibur (Becton Dickinson, San Diego, California, USA) using a panel of antibodies: CD45, CD14, surface CD3, cytoplasmic CD3, CD4, CD5, CD7, CD8, CD16+56, CD19, CD103, CD13, CD25, HLA-DR, CD30, TCR-alpha/beta and TCR-gamma/delta (BD Bioscience). Data were analysed using Cellquest software (Becton Dickinson).

\section{Histopathological assessment}

SB biopsies (Hematoxylin \& Eosin stained) were classified as (1) absence of VA and no increase in IELs (normal), (2) increased IELs without VA (Marsh I-II) and (3) increased IELs with partial to total VA (Marsh IIIa-IIIc). ${ }^{15}$

\section{Disease classification}

Patients were classified using currently recommended clinicopathological criteria. ${ }^{16}$ Newly diagnosed patients with $C D$ were designated as having active $\mathrm{CD}(\mathrm{ACD})$. The diagnosis was based on serological and histopathological findings, while patients were on a gluten-containing diet. Patients that did not exhibit VA (Marsh I/II) but had positive serologies and symptom response to GFD were included in this category. Patients on GFD following the diagnosis of $\mathrm{CD}$ were classified as GFD. Patients with persistent/recurrent malabsorptive symptoms with VA despite being on GFD for at least 12 months, after excluding other aetiologies or any malignancy, were classified as RCD. These cases were subdivided into RCDI and RCDII based on the absence or presence of phenotypically aberrant IELs, respectively, assessed by FC. Patients whose clinical, biopsy and serological findings did not support a diagnosis of $\mathrm{CD}$ or those diagnosed with other gastrointestinal disorders were classified as non-CD.

\section{Statistical analysis}

Descriptive statistics are presented for disease classification and results of TCR-GR analysis. The demographic, clinical, flow cytometric and histological characteristics of the patient groups were compared using Fisher's exact test, $\mathrm{X}^{2}$ test for categorical variables, and the t-test and analysis of variance test for non-parametric variables as appropriate.

\section{RESULTS}

\section{Case composition}

During the study period, 286 samples (from 245 patients) were identified. Sixteen samples from patients with incomplete clinical information $(n=12)$ and those with non-CD related T cell lymphoproliferative disorders $(n=4)$ were excluded. The distribution of the remaining 270 samples was as follows: 187 GFD (172 patients), eight ACD (eight patients), 51 RCDI (33 patients), nine RCDII (three patients) and 15 non-CD (14 patients). Samples from a single time point were used for TCR-GR analysis in 202 patients (88\%) and 28 patients had repeat biopsies analysed, the majority for persistent symptoms (table 1). Data of nine patients diagnosed with RCDI elsewhere prior to the 
Table 1 Clinicopathological characteristics of patients at the time of first analysis of small bowel biopsies

\begin{tabular}{|c|c|c|c|c|c|c|c|}
\hline & Total $(n=221)$ & $\operatorname{ACD}(n=8)$ & GFD (n=172) & RCDI $(n=24)$ & RCDII $(n=3)$ & Non-CD $(n=14)$ & $P$ values \\
\hline $\begin{array}{l}\text { Age (mean (SE)) } \\
\text { (range) }\end{array}$ & & $\begin{array}{l}44.25(5.1) \\
(26-71)\end{array}$ & $\begin{array}{l}48.88(1.3) \\
(19-88)\end{array}$ & $\begin{array}{l}57.38(2.9) \\
(24-79)\end{array}$ & $\begin{array}{l}67.00(5.1) \\
(42-75)\end{array}$ & $\begin{array}{l}60.86(3.4) \\
(40-76)\end{array}$ & 0.0052 \\
\hline Gender & & & & & & & 0.7946 \\
\hline Female & 169 & 7 & 131 & 18 & 3 & 10 & \\
\hline Male & 52 & 1 & 41 & 6 & 0 & 4 & \\
\hline $\begin{array}{l}\text { Duration of disease mean (SE) } \\
\text { [range], in months }\end{array}$ & & $1.8(1.1)(0-6)$ & $\begin{array}{l}89.2(12.4)(2- \\
1251)\end{array}$ & $\begin{array}{l}105.2(25.8)(8- \\
655)\end{array}$ & 84.00 & $9.6(2.6)(4-18)$ & $0.4811\left(0.3248^{*}\right)$ \\
\hline Diarrhoea & & $2 / 8(25 \%)$ & $102 / 172(59 \%)$ & $15 / 24(63 \%)$ & $1 / 3(33 \%)$ & $8 / 14(57 \%)$ & 0.3336 \\
\hline Immunomodulatory therapy & & $0 / 6(0 \%)$ & $23 / 158(16 \%)$ & $13 / 23(57 \%)$ & $2 / 2(100 \%)$ & 4/11 (42\%) & $<0.0001$ \\
\hline Positive serology & & $6 / 8(75 \%)$ & $64 / 150(43 \%)$ & $6 / 23(26 \%)$ & $2 / 3(67 \%)$ & $0 / 12(0 \%)$ & $0.0049(0.0967 \dagger)$ \\
\hline Histology & & & & & & & $<0.0001(0.0179 \ddagger)$ \\
\hline VA with $\uparrow$ IELs & & $6 / 8(75 \%)$ & $51 / 172(30 \%)$ & $24 / 24(100 \%)$ & $3 / 3(100 \%)$ & $7 / 14(50 \%)$ & \\
\hline 个IELs without VA & & $2 / 8(25 \%)$ & $54 / 172(31 \%)$ & $0 / 24(0 \%)$ & $0 / 3(0 \%)$ & $1 / 14(7 \%)$ & \\
\hline Normal & & $0 / 8(0 \%)$ & 67/172 (39\%) & $0 / 24(0 \%)$ & $0 / 3(0 \%)$ & $6 / 14(43 \%)$ & \\
\hline TCR & & & & & & & $0.0044(0.3888 \S)$ \\
\hline Clonal & & $0 / 8(0 \%)$ & $11 / 172(6 \%)$ & $4 / 24(17 \%)$ & $2 / 3(67 \%)$ & $0 / 14(0 \%)$ & \\
\hline Polyclonal & & $7 / 8(88 \%)$ & $130 / 172(76 \%)$ & $14 / 24(58 \%)$ & $0 / 3(0 \%)$ & $10 / 14(71 \%)$ & \\
\hline PCP & & $1 / 8(12 \%)$ & $31 / 172(18 \%)$ & $6 / 24(25 \%)$ & $1 / 3(33 \%)$ & $4 / 14(29 \%)$ & \\
\hline
\end{tabular}

${ }^{*}$ Analysis of GFD, RCDI and RCDII groups only.

†Analysis of ACD, GFD, RCDI and RCDII groups only.

$¥$ Analysis of non- $R C D$ disease categories only.

$\S$ Analysis of $A C D, G F D, R C D I$ and non-CD groups only.

$A C D$, active coeliac disease; GFD, gluten-free diet; IELs, intraepithelial lymphocytes; non-CD, aetiology other than coeliac disease; PCP, prominent clonal peaks; RCD, refractory

coeliac disease; TCR, T cell receptor rearrangement; VA, villous atrophy; $\mathrm{SE}$, standard error.

commencement of our study, with only follow-up biopsies analysed at our institution, are excluded from table 1 (please see the section Analysis of follow-up biopsies below for details).

\section{Patient demographics and clinicopathological characteristics}

Clinical and pathological characteristics of patients at the time of first biopsy during the study period are shown in table 1 . Consistent with prior observations, our series showed a female predominance $(76 \%)$. There was no statistical difference in the gender ratio and type of presenting symptoms between disease groups. The mean age of patients with RCDI and RCDII at the time of biopsy was significantly higher than that of patients with ACD and GFD ( $p=0.005)$. Patients in the ACD and non-CD groups had shorter disease duration (mean 1.8 and 9.6 months, respectively). However, no significant difference in disease duration was observed between GFD, RCDI and RCDII groups $(p=0.32)$. A significantly higher number of patients with RCDI and RCDII received immunomodulatory therapy (prednisone, budesonide and/or azathioprine) compared with those on GFD ( $\mathrm{p}<0.0001)$; none of the patients with ACD received such therapy. VA was significantly more frequent in patients with ACD compared with patients on GFD and non-CD patients $(p=0.018)$. Diagnoses of patients in the non-CD group comprised collagenous sprue $(n=2)$, tropical sprue $(n=3)$ and eosinophilic oesophagitis $(\mathrm{n}=1)$, all showing VA, and gastro-oesophageal reflux disease and non-coeliac gluten sensitivity $(n=1$, each), both lacking VA. Six patients did not have a specific pathological diagnosis and all except one showed normal histology.

\section{Patients with ACD}

In the ACD cohort (eight samples from eight patients), none of the SB biopsies showed clonal TCR-GR products. One case showed a PCP pattern, while biopsies from seven patients $(87.5 \%)$ showed polyclonal products (table 1$)$.

\section{Patients on GFD}

One hundred and eighty seven samples from 172 patients were analysed. A polyclonal TCR-GR pattern was seen in 130/172 (76\%) patients and $31(18 \%)$ and $11(6 \%)$ showed PCP and clonal TCR-GR patterns, respectively. Positive serologies and/or VA were detected in 5/11 (45\%) patients with clonal TCR-GRs and $16 / 31(52 \%)$ patients with a PCP pattern. Of patients with both negative serology and normal histopathology, 1/41 (2.4\%) had a clonal TCR-GR and 4/41 (9.8\%) had a PCP pattern (table 1).

\section{Patients with RCDI}

Fifty-one samples from 33 patients were analysed. As mentioned above, RCDI was diagnosed elsewhere in nine patients and only follow-up biopsies (showing normal histology) were assessed at our institution (see the Analysis of follow-up biopsies section below).

All 24 patients diagnosed with RCDI on first biopsy within the study period showed VA; 63\% had diarrhoea-predominant symptoms and $57 \%$ received immunomodulatory drug therapy. Clonal TCR-GR was detected in 4/24 (17\%) patients and 3/4 $(75 \%)$ had positive serologies. The PCP pattern was seen in $6 / 24$ (25\%) patients, none with positive serologies. Of the 14 patients with polyclonal products, three $(21 \%)$ had positive serologies (table 1).

Of six RCDI patients with positive serology, three (50\%) displayed clonal TCR-GR and three $(50 \%)$ had polyclonal TCR products. Of 17 patients with negative serology, one (6\%) displayed clonal TCR-GR, six (35\%) exhibited a PCP pattern and $10(59 \%)$ showed polyclonal products.

\section{Patients with RCDII}

Nine biopsies were evaluated from three patients. All three patients had follow-up biopsies during the study period and all 
Table 2 Follow-up clinicopathological data of cases with initial clonal or PCP TCR GR pattern

\begin{tabular}{|c|c|c|c|c|c|c|c|c|c|c|c|c|c|}
\hline & \multirow{2}{*}{$\begin{array}{l}\text { TCR pattern } \\
\text { (initial } \rightarrow \text { f/u) }\end{array}$} & \multicolumn{2}{|c|}{ Disease status } & \multirow{2}{*}{$\begin{array}{l}\text { Interval, } \\
\text { months }\end{array}$} & \multicolumn{2}{|c|}{ Histology } & \multicolumn{2}{|c|}{ Symptoms } & \multicolumn{2}{|c|}{ Serology } & \multirow{2}{*}{$\begin{array}{l}\text { CD4(-) } \\
\text { CD8(-) }\end{array}$} & \multirow[b]{2}{*}{ sCD3(-) } & \multirow[b]{2}{*}{ TCR (-) } \\
\hline & & At $d x$ & $f / u$ & & At $d x$ & $f / u$ & At $d x$ & $f / u$ & At $d x$ & $f / u$ & & & \\
\hline & \multicolumn{13}{|l|}{ Transient } \\
\hline 1 & $\mathrm{C} \rightarrow \mathrm{PC}$ & GFD & GFD & 24 & 个IEL & VA & Absent & Absent & + & - & $<20$ & $<10$ & $<10$ \\
\hline 2 & $\mathrm{PCP} \rightarrow \mathrm{PC}$ & GFD & GFD & 6 & 个IEL & TIEL & Absent & Present & - & - & $27-34$ & $<10$ & $<10$ \\
\hline 3 & $\mathrm{PCP} \rightarrow \mathrm{PC}$ & GFD & GFD & 23 & VA & VA & Present & Absent & + & - & $<20$ & $<10$ & $<10$ \\
\hline \multirow[t]{2}{*}{4} & $\mathrm{PCP} \rightarrow \mathrm{PC}$ & GFD & GFD & 12 & 个IEL & 个IEL & Present & Present & - & - & $<20$ & $<10$ & $<10$ \\
\hline & Persistent & & & & & & & & & & & & \\
\hline 5 & $\mathrm{PCP} \rightarrow \mathrm{C}(\neq)$ & RCDI & RCDI & 12 & VA & VA & Present & Present & - & - & $<20$ & $<10$ & $<10$ \\
\hline 6 & $\mathrm{PCP} \rightarrow \mathrm{PCP}(=)$ & RCDII & RCDII & 6 & VA & VA & Absent & Absent & + & $?$ & $1-21$ & $69-70$ & $<10$ \\
\hline 7 & $\mathrm{PCP} \rightarrow \mathrm{PCP}(=)$ & RCDI & RCDI & 20 & VA & VA & Present & Present & - & + & $<20$ & $<10$ & $<10$ \\
\hline 8 & $\mathrm{PCP} \rightarrow \mathrm{PCP}(=)$ & RCDI & RCDI & 23 & VA & VA & Present & Present & - & - & $<20$ & $<10$ & $2-13$ \\
\hline 9 & $\mathrm{PCP} \rightarrow \mathrm{C}(=)$ & RCDI & RCDI & 25 & 个IEL & VA & Absent & Absent & - & - & $16-25$ & $<10$ & $<10$ \\
\hline 10 & $\mathrm{C} \rightarrow \mathrm{PCP}(=)$ & GFD & GFD & 15 & VA & VA & Absent & Absent & + & - & $<20$ & $<10$ & $<10$ \\
\hline 11 & $\mathrm{PCP} \rightarrow \mathrm{PC} \rightarrow \mathrm{PCP}(=)$ & RCDI & RCDI & 9,14 & VA & VA & Absent & Present & - & + & $30-46$ & $<10$ & $<10$ \\
\hline 12 & $C \times 3(=)$ & RCDII & RCDII & 12,18 & 个IEL & 个IEL & Present & Present & - & $?$ & $20-28$ & $17-26$ & $<10$ \\
\hline 13 & $C \times 5(=)$ & RCDII & RCDII & $9,2,7,5$ & VA & VA & Absent & Present & - & - & $<20$ & $36-85$ & $<10$ \\
\hline
\end{tabular}

C, clonal; dx, diagnosis; f/u, follow-up; GR, gene rearrangement; IEL, intraepithelial lymphocytes; PC, polyclonal; PCP, prominent clonal peaks; TCR, T cell receptor; VA, villous atrophy; ?, unknown; (=), identical size products; (\#), different size products.

received immunomodulatory drug therapy. Two patients showed clonal TCR-GR and one displayed the PCP pattern (table 1). The latter case was diagnosed as RCDII prior to the study period based on histopathological features, detection of aberrant phenotype by FC and clonal products by TCR-GR analysis.

\section{Analysis of follow-up biopsies}

Thirteen patients with GFD had follow-up biopsies, with three (23\%) subsequently being diagnosed as RCDI. Two of these cases demonstrated no change in the TCR pattern (polyclonal), whereas one showed a PCP pattern on repeat biopsy.

Twelve patients with RCDI had follow-up biopsies, including nine that were first diagnosed elsewhere. None showed disease progression to RCDII, with $7 / 12$ cases $(58 \%)$ remaining polyclonal on repeat testing and five (42\%) showing persistent clonal or PCP TCR-GR patterns.

All three patients with RCDII had follow-up biopsies, with two showing persistent clonal TCR-GRs in multiple repeat biopsy samples $(\mathrm{n}=8)$ and one showing persistent PCP pattern and VA.

Altogether, of the 28 patients with follow-up biopsies, three $(11 \%)$ had a change in diagnosis from GFD to RCDI (interval between biopsies 11-32 months). Diagnosis was unchanged on repeat biopsy in 25 patients (interval between biopsies 5-35 months).

In 13/28 (46\%) patients with repeat biopsies, clonal $(\mathrm{n}=4$, $31 \%)$ or PCP $(n=9,69 \%)$ TCR-GR patterns were found at first biopsy in our study period with none showing disease progression (table 2). A 'transient' clonal $(\mathrm{n}=1)$ or PCP $(\mathrm{n}=3)$ TCR-GR pattern was noted in 4/13 cases (all GFD) with follow-up biopsies showing a polyclonal TCR-GR pattern (time interval between biopsies 6-24 months). Two of these showed VA and positive serology suggesting exposure to dietary gluten. Persistent clonal or PCP TCR-GR patterns were observed in 9/13 patients (time interval between biopsies 5-25 months). Identical sized clonal product or PCPs were found on subsequent biopsies in $8 / 9$ (89\%) patients, including three with RCDII, with the majority exhibiting VA and a subset $(\mathrm{n}=4,50 \%)$ demonstrating positive serologies. One patient with RCDI showed different clonal/PCP TCR-GR patterns on repeat biopsies with persistent VA.
In the remaining $15 / 28$ patients with repeat biopsies, 13 showed polyclonal TCR-GR on initial and follow-up biopsies with no change in diagnosis and 2/15 patients demonstrated polyclonal TCR-GR on initial diagnosis with follow-up biopsies showing a clonal or PCP pattern. The latter case progressed from GFD to RCD1 while the former showed no disease progression.

\section{Concurrent $T C R-\beta$ GR analysis of fresh and FFPE biopsy samples}

Repeat TCR-GR analysis of FFPE biopsies from the same time point in $57 / 71(80 \%)$ cases confirmed the original TCR-GR pattern, while $14(20 \%)$ showed discordant results, findings in line with prior studies. ${ }^{17}$ Seven $(10 \%)$ showed conversion of PCP pattern to polyclonal or vice versa and were considered to represent 'pseudoclones' and 6 (8\%) showed clonal TCR-GR on analysis of fresh biopsy and a PCP pattern on FFPE or vice versa with unchanged TCR-GR product size. These cases were considered to harbour clonal TCR-GRs. One case with a polyclonal TCR-GR on analysis of fresh biopsy showed clonal TCR-GR on testing FFPE tissue. This patient was also considered to have clonal TCR-GR for the purpose of this study. The follow-up biopsy of this patient (GFD) showed polyclonal TCR-GR with no change in diagnosis.

\section{Correlation of $T C R-\beta$ GR patterns with clinical and pathological features}

Overall, a significantly higher proportion of patients with RCDII showed clonal TCR-GR compared with other disease groups $(p=0.004)$. There was no significant difference in the frequencies of different TCR-GR patterns between the non-RCDII disease groups $(p=0.39)$. Clonal TCR-GR was found in $6 \%$ of GFD and $17 \%$ of RCDI, but in none of the ACD and non-CD cases. The PCP pattern was seen in all patient groups including 4 cases in the non-CD group, without significant differences between the groups (table 1).

Analysis of the cases stratified by TCR-GR patterns (clonal, polyclonal and PCP) showed no significant differences between the groups with regard to age, clinical, serological and histological parameters (table 3). Specifically, comparison of the 
Table 3 Clinicopathological, molecular and phenotypic characteristics of cases with different TCR-GR patterns

\begin{tabular}{|c|c|c|c|c|}
\hline & Clonal & Polyclonal & PCP & $P$ values \\
\hline $\begin{array}{l}\text { Age mean (SE) } \\
\text { (range) }\end{array}$ & $\begin{array}{l}56.29(3.8) \\
(31-88)\end{array}$ & $\begin{array}{l}50.07(1.3) \\
(19-86)\end{array}$ & $\begin{array}{l}50.88(2.8) \\
(19-81)\end{array}$ & 0.3676 \\
\hline Histology & & & & 0.4380 \\
\hline VA & $9 / 17(53 \%)$ & $65 / 161(40 \%)$ & $17 / 43(40 \%)$ & \\
\hline 个IELs without VA & $5 / 17(29 \%)$ & $38 / 161(24 \%)$ & $14 / 43(32 \%)$ & \\
\hline Normal & $3 / 17(18 \%)$ & $58 / 161(36 \%)$ & $12 / 43(28 \%)$ & \\
\hline Positive serology & $7 / 14(50 \%)$ & $57 / 141(40 \%)$ & $15 / 42(36 \%)$ & 0.7373 \\
\hline Diarrhoea & 9/17 (53\%) & $97 / 163(60 \%)$ & $23 / 43(53 \%)$ & 0.7091 \\
\hline Immunomodulatory therapy & $5 / 17(29 \%)$ & $32 / 149(21 \%)$ & $7 / 36(19 \%)$ & 0.7031 \\
\hline TCR PCR primer sets & & & & $0.5081^{*}$ \\
\hline D-J & $5 / 17(30 \%)$ & 0/163 & $19 / 43(44 \%)$ & \\
\hline V-J & $6 / 17(35 \%)$ & 0/163 & $10 / 43(23 \%)$ & \\
\hline D-J and V-J & $6 / 17(35 \%)$ & $0 / 163$ & $14 / 43(33 \%)$ & \\
\hline \multicolumn{5}{|l|}{ Flow (\%) mean (SE)(range) } \\
\hline $\mathrm{CD} 8(+)$ & $56.35(7)(2-90)$ & $60.99(1.2)(5-94)$ & $59.37(2.5)(13-82)$ & 0.5259 \\
\hline $\mathrm{CD} 4(+)$ & $13.94(3.6)(4-57)$ & $15.46(1.0)(2-82)$ & $16.28(1.8)(4-67)$ & 0.8022 \\
\hline TCR a/b & $62.82(6.2)(10-90)$ & $64.52(1.1)(33-93)$ & $63.76(2.5)(13-92)$ & 0.8929 \\
\hline $\mathrm{TCR} \mathrm{g} / \mathrm{d}$ & $13.06(2.9)(1-38)$ & $20.37(1.1)(1-62)$ & $19.17(2.3)(1-56)$ & 0.1359 \\
\hline TCR(-) & $0.47(2.0)(-23$ to 18$)$ & $3.14(0.5)(-12$ to 43$)$ & $4.24(1.2)(-23$ to 29$)$ & 0.1630 \\
\hline $\mathrm{sCD} 3(-)$ & $17.8(8.2)(-2$ to 86$)$ & $2.77(0.4)(-14$ to 24$)$ & $3.41(1.9)(-12$ to 70$)$ & $<0.0001(0.0227 t, 0.6141 \ddagger)$ \\
\hline
\end{tabular}

*Analysis of clonal and PCP groups only.

tAnalysis excluding the RCDII group.

¥Analysis of polyclonal and PCP groups only.

GR, gene rearrangement; IELs, intraepithelial lymphocytes; PCP, prominent clonal peaks; RCDII, refractory coeliac disease type II; TCR, T cell receptor rearrangement; VA, villous atrophy; SE, standard error.

clonal and PCP groups showed no significant differences in the frequency of VA, serological status, diarrhoea-predominant presentation and use of immunomodulatory drug therapy. However, analysis of FC showed a significantly higher fraction of surface CD3(-) lymphocytes in the clonal TCR-GR group compared with the PCP and polyclonal groups $(\mathrm{p}<0.0001)$. These results were significant even when RCDII cases were excluded from analysis $(p=0.023)$. There was no association between cases with clonal TCR-GR and expansions of other $\mathrm{T}$ cell populations. Additionally, no statistically significant differences were observed between the clonal and PCP cases with respect to the PCR primer sets showing abnormalities (D-J and/ or $\mathrm{V}-\mathrm{J}$ ) (table 3).

Furthermore, no statistically significant differences in the TCR-GR patterns of GFD cases with and without VA were observed, irrespective of the duration of GFD $(p=0.91)$ or on comparing patients on GFD for $>12$ months to those on GFD $<12$ months from diagnosis $(p=0.9)$ (see online Supplementary table S1). No significant differences in the TCR-GR patterns were noted on comparing seropositive and seronegative patients with GFD $(p=0.91)$. Analysis of TCR-GR patterns based on the presence or absence of VA, when ACD and GFD groups were combined, also did not show any statistically significant differences $(p=0.88)$ (online Supplementary table S1).

\section{DISCUSSION}

RCDI and II are rare complications of CD that have significantly different clinical features and prognoses. ${ }^{16}$ Current diagnostic algorithms incorporate the IEL phenotype and results of TCR-GR analyses, in distinguishing between RCDI and II. ${ }^{4}$ The phenotype of IELs in CD and RCDI and II has been reasonably well characterised. ${ }^{12}{ }^{18-20}$ However, data regarding the patterns of TCR-GR in different phases or clinical presentations of $\mathrm{CD}$ are limited and their clinical significance remains unclear. ${ }^{11-14}$ Standardisation of multiplex PCR assays and optimisation of primers for TCR-GR studies has led to increased sensitivity in the detection of clonal $\mathrm{T}$ cell populations in $\mathrm{T}$ cell neoplasms. ${ }^{10}$ In our study, we investigated the significance of the different TCR-GR patterns using the BIOMED-2 primers in different phases of $\mathrm{CD}$, including RCD, and correlated these findings with the relevant clinical and pathological features.

We found that the presence of clonal TCR-GR is not specific for RCDII. This pattern was seen in a small but significant fraction of RCDI and GFD cases (17\% and 6\%, respectively), but not in ACD and non-CD cases. However, PCPs were detected in a sizeable subset of cases in all disease groups, including RCDII. There was no association between clonal or PCP TCR-GR patterns and the clinicopathological characteristics of CD patients assessed in this study. This finding supports the results of prior studies of RCD patients, which found no association between detection of clonal TCR-GR in SB biopsies and clinical outcome. ${ }^{21}{ }^{22}$ Intriguingly, FC showed an association between a significantly higher $\mathrm{sCD} 3(-)$ lymphocytic population and clonal TCR-GR in non-RCDII SB biopsies. The significance of this finding is unclear. FC analysis used in this study could not reliably distinguish whether the sCD3(-) lymphocytes represented activated $\mathrm{T}$ cells that had downregulated CD3 or intraepithelial innate lymphoid cells (ILCs), which lack sCD3 expression. $^{2324}$ As no increase in sCD3(-) lymphocytes was seen in ACD biopsies, we favour these cells to be ILCs. Importantly, since whole biopsy samples were used for FC and TCR-GR analysis, the source of clonal TCR-GRs (lamina propria T cells vs IELs) is not known. Future analyses of purified or sorted lymphocyte fractions are awaited to discern if clonal TCR-GRs reflect expansions of intraepithelial innate $\mathrm{T}$ cells or ILCs or gliadin-responsive lamina propria $T$ cells.

In a subset of our cases with follow-up biopsies, persistence of identical-sized PCR products (either as a distinct clone or 
PCP) was noted for over 2 years without evidence of RCDII based on FC phenotyping of the IELs. Nearly all of these cases showed persistent VA and half of the non-RCDII cases showed positive serologies consistent with gluten exposure. Our findings somewhat differ from those of Liu and colleagues, ${ }^{11}$ who detected clonal TCR-GRs in $16 \%$ of CD cases. Transient clonal GRs were associated with GFD-non-compliance but not with expansions of aberrant IELs. However, persistent clonal TCR-GRs detected in 9\% of CD cases were associated with concurrent or progressive expansion of aberrant IELs and progression to RCDII or EATL. Of interest, persistence of aberrant IELs or clonal TCR-GR alone was not associated with development of EATL. In their study, aberrant IELs were quantified by immunohistochemistry and a cut-off value of $40 \%$ was established to diagnose RCDII. It has been shown that immunohistochemistry is suboptimal for phenotypic characterisation of IELs and this modality can misclassify a proportion of CD or RCDI cases as RCDII. ${ }^{18} 1920$ In our study, FC analysis allowed precise characterisation of IELs and a more accurate assessment of disease status.

A recent study utilising high-throughput sequencing of TCR- $\beta$ genes to characterise the small intestinal T cell repertoire in CD and RCD detected frequent dominant clonotypes in RCDII cases along with lower numbers of other clonotypes (reduced repertoire). ${ }^{25}$ Dominant TCR sequences/clonotypes were also noted in the biopsies of patients without $\mathrm{CD}$ and with RCDI, while patients with ACD had a significantly higher number of clonotypes (expanded repertoire) compared with CD patients on GFD, which might reflect bystander activation and expansion of $\mathrm{T}$ cells as a consequence of inflammation. The dominant clones in RCDII appeared gliadin-independent, and the authors found that $4 / 5$ patients with RCDII that eventually progressed to EATL showed $>50 \%$ frequency of the most common clonotypes. However, similar to our findings, persistence of specific TCR clones was noted in patients with GFD (and ACD) for several years without disease progression to RCDII. Further studies evaluating the relationship of specific TCR clonotypes with expansions of aberrant IELs may help elucidate the pathogenesis of RCDII.

Comprehensive evaluation of GFD adherence is crucial when assessing for RCD. This can be challenging in symptomatic patients who report strict dietary compliance and are unaware of surreptitious/trace gluten ingestion. ${ }^{4} 2627$ Furthermore, there is variability among patients with regard to gluten tolerance and time to normalisation of the duodenal mucosa following GFD. ${ }^{48}{ }^{29}$ Characterisation of TCR-GRs by next-generation sequencing to determine the composition of $\mathrm{T}$ cell clonotypes in patients on stringent GFD and those consuming gluten might be useful in understanding the impact of low level gluten exposure on TCR-GR assays.

Our study indicates that the results of TCR-GR analyses should be interpreted with caution when entertaining a diagnosis of RCDII, as clonal TCR-GRs can be seen in up to $17 \%$ of non-RCDII cases. Corroboration with the results of FC determined IEL phenotype and biopsy histopathology is necessary. The PCP pattern, observed in $12 \%-29 \%$ of non-RCDII biopsies, does not define clonality and can be seen in all phases of CD as well as in non-CD cases. Since discrepant TCR-GR results between fresh (or frozen) and FFPE samples have been described and also noted in 20\% of our cases, analysis of fresh or frozen biopsies is recommended. ${ }^{17}$ However, repeat TCR-GR analysis on a different FFPE biopsy sample or a follow-up biopsy may help clarify an indeterminate result. ${ }^{10} 30$ The complexity these analyses
Take home messages

- Clonal T cell receptor gene rearrangements in small intestinal biopsies are not specific for refractory coeliac disease type II (RCDII) and can be seen in up to $17 \%$ of non-RCDII cases, including patients with uncomplicated coeliac disease (CD) on gluten-free diet and RCDI.

- AT cell receptor gene rearrangement pattern showing prominent clonal peaks in a polyclonal background can be observed in all phases of $C D$ and does not necessarily indicate the presence of a clonal $\mathrm{T}$ cell population.

- Persistence of identical-sized clonal products or prominent clonal peaks is not uncommon in $\mathrm{CD}$; hence, this phenomenon by itself should not be considered evidence of RCDII.

- Corroboration of the T cell receptor gene rearrangement findings with results of flow cytometry to evaluate intraepithelial lymphocyte (IEL) phenotype and small intestinal biopsy histopathology is necessary for accurate diagnosis and classification of RCD.

present for evaluating suspected RCDII suggests that patients are best served by specialised centres that employ a multidisciplinary approach to the diagnosis and clinical management of patients with CD.

\section{Handling editor Runjan Chetty.}

Contributors PHG and GB were responsible for the study concept and design. SH, TG, CAG, MM, PHG and GB were responsbile for the acquisition of data. TG, SH and $G B$ were involved in the analysis and interpretation of data and drafting the manuscript. TG and SH did the statistical analysis. All the authors contributed to the critical revision of the manuscript for important intellectual content.

Funding The authors have not declared a specific grant for this research from any funding agency in the public, commercial or not-for-profit sectors.

Competing interests None declared.

Patient consent Not required.

Ethics approval Institutional Review Board of Columbia University, New York, USA.

Provenance and peer review Not commissioned; externally peer reviewed.

(c) Article author(s) (or their employer(s) unless otherwise stated in the text of the article) 2018. All rights reserved. No commercial use is permitted unless otherwise expressly granted.

\section{REFERENCES}

1 Rubio-Tapia A, Murray JA. Classification and management of refractory coeliac disease. Gut 2010;59:547-57.

2 Lionetti E, Catassi C. New clues in celiac disease epidemiology, pathogenesis, clinical manifestations, and treatment. Int Rev Immunol 2011;30:219-31.

3 Daum S, Cellier C, Mulder CJ, et al. Refractory coeliac disease. Best Pract Res Clin Gastroenterol 2005;19:413-24.

4 Nijeboer P, van Wanrooij RL, Tack GJ, et al. Update on the diagnosis and management of refractory coeliac disease. Gastroenterol Res Pract 2013;2013:1-9.

5 Di Sabatino A, Biagi F, Gobbi PG, et al. How I treat enteropathy-associated T-cell lymphoma. Blood 2012;119:2458-68.

6 Woodward J. Improving outcomes of refractory celiac disease - current and emerging treatment strategies. Clin Exp Gastroenterol 2016;9:225-36.

7 van de Water JM, Cillessen SA, Visser OJ, et al. Enteropathy associated T-cell lymphoma and its precursor lesions. Best Pract Res Clin Gastroenterol 2010;24:43-56.

8 van Wanrooij RL, Müller DM, Neefjes-Borst EA, et al. Optimal strategies to identify aberrant intra-epithelial lymphocytes in refractory coeliac disease. J Clin Immunol 2014;34:828-35

9 Perfetti V, Brunetti L, Biagi F, et al. TCR $\beta$ clonality improves diagnostic yield of TCR $\gamma$ clonality in refractory celiac disease. J Clin Gastroenterol 2012;46:675-9.

10 van Dongen JJ, Langerak AW, Brüggemann $\mathrm{M}$, et al. Design and standardization of PCR primers and protocols for detection of clonal immunoglobulin and T-cell receptor 
gene recombinations in suspect lymphoproliferations: report of the BIOMED-2 Concerted Action BMH4-CT98-3936. Leukemia 2003;17:2257-317.

11 Liu H, Brais R, Lavergne-Slove A, et al. Continual monitoring of intraepithelial lymphocyte immunophenotype and clonality is more important than snapshot analysis in the surveillance of refractory coeliac disease. Gut 2010;59:452-60.

12 Cellier C, Patey N, Mauvieux L, et al. Abnormal intestinal intraepithelial lymphocytes in refractory sprue. Gastroenterology 1998;114:471-81.

13 Daum S, Weiss D, Hummel M, et al. Frequency of clonal intraepithelial T lymphocyte proliferations in enteropathy-type intestinal T cell lymphoma, coeliac disease, and refractory sprue. Gut 2001;49:804-12.

14 Ubiali A, Villanacci V, Facchetti F, et al. Is TCR gamma clonality assay useful to detect early celiac disease? J Clin Gastroenterol 2007:41:275-9.

15 Oberhuber G, Granditsch G, Vogelsang H. The histopathology of coeliac disease: time for a standardized report scheme for pathologists. Eur J Gastroenterol Hepatol 1999:11:1185-94.

16 Ludvigsson JF, Bai JC, Biagi F, et al. Diagnosis and management of adult coeliac disease: guidelines from the British Society of Gastroenterology. Gut 2014;63:1210-28

17 Christensen M, Funder AD, Bendix K, et al. Comparative investigations of T cell receptor gamma gene rearrangements in frozen and formalin-fixed paraffin waxembedded tissues by capillary electrophoresis. J Clin Pathol 2006;59:645-54 .

18 Cellier C, Delabesse E, Helmer C, et al. Refractory sprue, coeliac disease, and enteropathy-associated T-cell lymphoma. French Coeliac Disease Study Group. Lancet 2000;356:203-8.

19 Sanchez-Muñoz LB, Santón A, Cano A, et al. Flow cytometric analysis of intestinal intraepithelial lymphocytes in the diagnosis of refractory celiac sprue. Eur J Gastroenterol Hepatol 2008;20:478-87.
20 van Wanrooij RL, Schreurs MW, Bouma G, et al. Accurate classification of RCD requires flow cytometry. Gut 2010;59:59.

21 Malamut G, Afchain P, Verkarre V, et al. Presentation and long-term follow-up of refractory celiac disease: comparison of type I with type II. Gastroenterology 2009;136:81-90

22 Arguelles-Grande C, Brar P, Green PH, et al. Immunohistochemical and T-cell receptor gene rearrangement analyses as predictors of morbidity and mortality in refractory celiac disease. J Clin Gastroenterol 2013;47:593-601.

23 Schmitz F, Kooy-Winkelaar Y, Wiekmeijer AS, et al. The composition and differentiation potential of the duodenal intraepithelial innate lymphocyte compartment is altered in coeliac disease. Gut 2016;65:1269-78.

24 Ettersperger J, Montcuquet N, Malamut G, et al. Interleukin-15-Dependent T-Cell-like Innate Intraepithelial Lymphocytes Develop in the Intestine and Transform into Lymphomas in Celiac Disease. Immunity 2016;45:610-25.

25 Ritter J, Zimmermann $\mathrm{K}$, Jöhrens $\mathrm{K}$, et al. T-cell repertoires in refractory coeliac disease. Gut 2018;67:gutjn-2016-311816.

26 Green PH, Cellier C. Celiac disease. N Engl J Med 2007:357:1731-43.

27 Hollon JR, Cureton PA, Martin ML, et al. Trace gluten contamination may play a role in mucosal and clinical recovery in a subgroup of diet-adherent non-responsive celiac disease patients. BMC Gastroenterol 2013;13:40.

28 Bruins MJ. The clinical response to gluten challenge: a review of the literature. Nutrients 2013:5:4614-41.

29 Biagi F, Campanella J, Martucci S, et al. A milligram of gluten a day keeps the mucosal recovery away: a case report. Nutr Rev 2004:62:360-3.

30 Langerak AW, Groenen PJ, Brüggemann M, et al. EuroClonality/BIOMED-2 guidelines for interpretation and reporting of Ig/TCR clonality testing in suspected lymphoproliferations. Leukemia 2012;26:2159-71. 